\title{
Legionella pneumophila induces human beta Defensin-3 in pulmonary cells
}

\author{
Stefanie Scharf1', Kremena Vardarova1', Friederike Lang ${ }^{1}$, Bernd Schmeck1,2, Bastian Opitz1 , Antje Flieger ${ }^{3}$, \\ Klaus Heuner' ${ }^{4}$ Stefan Hippenstiel', Norbert Suttorp' ${ }^{1}$ and Philippe D N'Guessan*1
}

\begin{abstract}
Background: Legionella pneumophila is an important causative agent of severe pneumonia in humans. Human alveolar epithelium and macrophages are effective barriers for inhaled microorganisms and actively participate in the initiation of innate host defense. The beta defensin-3 (hBD-3), an antimicrobial peptide is an important component of the innate immune response of the human lung. Therefore we hypothesize that hBD-3 might be important for immune defense towards L. pneumophila.

Methods: We investigated the effects of L. pneumophila and different TLR agonists on pulmonary cells in regard to hBD-3 expression by ELISA. Furthermore, siRNA-mediated inhibition of TLRs as well as chemical inhibition of potential downstream signaling molecules was used for functional analysis.

Results: L. pneumophila induced release of hBD-3 in pulmonary epithelium and alveolar macrophages. A similar response was observed when epithelial cells were treated with different TLR agonists. Inhibition of TLR2, TLR5, and TLR9 expression led to a decreased hBD-3 expression. Furthermore expression of hBD-3 was mediated through a JNK dependent activation of AP-1 (c-Jun) but appeared to be independent of NF-KB. Additionally, we demonstrate that hBD-3 elicited a strong antimicrobial effect on L. pneumophila replication.
\end{abstract}

Conclusions: Taken together, human pulmonary cells produce hBD-3 upon L. pneumophila infection via a TLR-JNK-AP1-dependent pathway which may contribute to an efficient innate immune defense.

\section{Background}

Legionella pneumophila is the causative agent of Legionnaires' disease, a severe pneumonia with high mortality [1]. The bacterium enters the human body by aerosol droplets and successfully establishes itself in macrophages and the alveolar epithelium, which normally offer an efficient barrier against infections [2]. Among the various putative virulence factors of this pathogen that have been identified to date, the type II (Lsp) and IVB (Dot/Icm) secretion system enable the bacteria to export proteins and therefore activates diverse cell signaling pathways [3,4]. Furthermore, bacterial cytoplasm membrane components, flagellin, and bacterial DNA, all major pathogen-associated factors of L. pneumophila, which activate innate immune response of alveolar epithelium as well as in macrophages [5-7]. L. pneumophila

* Correspondence: dje_philippe.nguessan@charite.de

1 Department of Internal Medicine/Infectious Diseases and Pulmonary Medicine, Charité - Universitätsmedizin Berlin, Germany

Full list of author information is available at the end of the article can be detected by means of toll-like receptors (TLR) or cytosolic pathogen pattern recognition receptors. Indeed it has been demonstrated that the atypical Legionella LPS can be recognized by TLR2, flagellin through TLR 5 and DNA via TLR9 [5-7]. To clear L. pneumophila from the lung, a functionally intact innate immune system must be present. There is increasing evidence that human $\beta$ defensins (hBDs), a family of endogenous, cationic antimicrobial and immunomodulatory peptides secreted at epithelial mucosal and macrophages are critical components of host defense $[8,9])$. The human $\beta$-defensin (hBD) family comprises multiple members. While hBD-1 is constitutively expressed [9], production of hBD-2 and hBD-3, can be induced upon stimulation with bacteria and/or cytokines [10-12]. hBD-3 is, unlike other hBDs, a saltinsensitive defensin with a broad antimicrobial activity against e.g. multidrug-resistant nosocomial strains [1013]. It has been reported that hBD-3 is expressed by pulmonary epithelial cells and increases in respiratory tract 
and serum of patients with bacterial pneumonia [14]. Consequently, the antibacterial properties of hBD-3 have attracted the attention of researchers in the field of pulmonary diseases.

Expression of hBD-3 is controlled by a tight regulatory network involving the transcription-factors Nuclear Factor- $\mathrm{kB}$ (NF- $\mathrm{kB}$ ) and the Activator Protein-1 (AP-1) [1517]. These transcription factors are activated by complex signalling pathways, including the JNK mitogen-activated protein kinase (MAPK) [18]. Although L. pneumophila efficiently infects and activates lung epithelial cells and alveolar macrophages [19-21], and hBD-3 secretion was observed in patients with bacterial pneumonia [14], regulatory mechanisms of $\mathrm{hBD}-3$ production in $L$. pneumophila infections is widely unknown.

In the study presented, we demonstrate that L. pneumophila induced hBD-3 in alveolar epithelium and macrophages. The hBD-3 expression was controlled by TLR2, TLR5 and TLR9, as well as activation of JNK and AP-1 (cJun) whereas NF- $\mathrm{KB}$ was not required. Also, recombinant hBD-3 elicited a strong antimicrobial effect on L. pneumophila. Moreover, inhibition of hBD-3 expression increased the L. pneumophila intracellular growth in pulmonary epithelium. Thus, hBD-3 production by pulmonary cells may contribute to the host response in Legionnaires' disease. Our results may significantly contribute to the understanding of the pathogenesis of Legionnaires' disease.

\section{Materials and methods Materials}

Recombinant human BD-3 was purchased from cellsciences (Canton, MA, USA). Erythromycin, Malp-2 (TLR2 ligand), ODN M362 (TLR9 ligand, a non-methylated CpG motif), and flagellin (TLR5 ligand) from Salmonella enterica serovar typhimurium were all purchased from Sigma Chem. Co. (Munich, Germany). TNF $\alpha$ were purchased from R\&D Systems (Wiesbaden, Germany). The JNK inhibitor (JNK II), the NF-kB inhibitor (NF-kB activator inhibitor) and MG-132 were all purchased from Calbiochem (Darmstadt, Germany). All other chemicals used were of analytical grade and obtained from commercial sources.

\section{Cell lines}

\section{Alveolar macrophages}

Human alveolar macrophages were obtained by bronchoalveolar lavages (BAL) of patients from routine diagnostic. The study was approved by the local ethics committee of the Charité-Universitätsmedizin Berlin in accordance with the ethical rules stated in the Declaration of Helsinki. Cells were recovered, washed twice in cold PBS, then resuspended at $106 / \mathrm{ml}$ in RPMI 1640 (PAA, Pasching, Austria) with $10 \%$ heat-inactivated FCS and antibiot- ics. Alveolar macrophages were placed into 24-well tissue culture plates and allowed to adhere for $2 \mathrm{~h}$. The monolayers were then washed three times to remove nonadherent cells and antibiotics, and cultured in RPMI 1640 with $10 \%$ heat-inactivated FCS until infections.

\section{Airway epithelial cells}

Primary human small airway epithelial cells (SAEC) were obtained from Cambrex (Cambrex, Taufkirchen, Germany) and cultured according to the supplier's instructions. The alveolar epithelial cell line A549 was purchased from DSMZ (Braunschweig, Germany) and cultured in HAM'S F 12 (PAA, Pasching, Austria) with L-glutamine, $10 \%$ FCS without antibiotics.

\section{Infection with bacterial strains}

L. pneumophila sg1 strain 130b (ATCC BAA-74, kindly provided by N. P. Cianciotto, Chicago, USA), strain JR32 (wild type), JR32 $\triangle \operatorname{dot} A$ deficient in $d o t / i c m$, encoding a protein essential for the type IVB secretion system (kindly provided by H. Shuman, New York, USA), strain Corby (wild type), Corby $\triangle$ flaA deficient in flagellin as well as its type II secretion system knock out Corby $\triangle l s p D E$ [3] were routinely grown on buffered charcoalyeast extract (BCYE) agar for 2 days at $37^{\circ} \mathrm{C}$ before used [22]. Heat inactivation of L. pneumophila was accomplished in a water bath at $56^{\circ} \mathrm{C}$ for $30 \mathrm{~min}$. No live bacteria were detected after this suspension was plated onto agar plates. The used cells were infected with L. pneumophila with a multiplicity of infection (MOI) of 10 at $37^{\circ} \mathrm{C}$ and $5 \% \mathrm{CO}_{2}$

\section{Replication assay}

To address the antimicrobial activity of hBD-3 towards $L$. pneumophila, $10^{5} \mathrm{cfu} / \mathrm{ml}$ bacteria were suspended in HAM's 12 without supplements and recombinant hBD-3 was added. Bacteria growth was enumerated by plating suspension on agar plates as colony-forming-units (cfu). For the intracellular replication assay, A549 cells were infected with L. pneumophila 130b. After $2 \mathrm{~h}$, cells were washed with PBS and remaining extracellular bacteria were killed by the cell-impermeable antibiotic gentamycin $(50 \mu \mathrm{g} / \mathrm{ml}$, Invitrogen, Karlsruhe, Germany) for $1 \mathrm{~h}$. Afterwards cells were washed twice with PBS (time point 0 ) and incubated in HAM's 12 medium without supplements with recombinant hBD-3. Intracellular bacteria were enumerated by lysed cell suspension plated on agar plates as cfu.

\section{Isolation of bacterial DNA}

Bacterial DNA from Legionella was prepared using a Qiagen construct kit protocol for endotoxin-free isolation of bacterial DNA (Qiagen, Hilden, Germany) as described elsewhere [23].

\section{RNA interference in A549 cells}

A549 cells were transfected by using Amaxa Nucleofector $^{\text {Tw }}$ (Amaxa, Köln, Germany) according to the manufacturer's protocol (Nucleofector $^{\text {Tw }}$ Solution V, Nucleofector ${ }^{\text {Tw }}$ 
program G-16) with $2 \mu \mathrm{g}$ siRNA per $10^{6}$ cells. Control non-silencing siRNA (sense UUCUCCGAACGUGUCACGUtt, antisense ACGUGACACGUUCGGAGAA), siRNA targeting TLR2 (sense GCACUUUAUAUUCACUUACtt, antisense GUAAGUGAAUAUAAAGUGCtc), TLR5 (sense GGAGCAAUUUCCAACUUAUtt, antisense AUAAGUUGGAAAUUGCUCCtt), and siRNA targeting TLR9 (sense CUGUCCUUCAAUUACCAAAtt antisense GUAAUUGAAGGACAGgt) were purchased from MWG (Ebersberg, Germany).

Polymerase chain reaction (RT-PCR)

Total RNA from cells was isolated with the RNeasy Mini kit (Qiagen, Hilden, Germany) and was reverse-transcribed using M-MLV reverse transcriptase (Invitrogen, Karlsruhe, Germany). The generated cDNA was amplified by semi-quantitative RT-PCR using RedTaq polymerase (Qiagen, Hilden, Germany). Primers specific for GAPDH (encoding glyceraldehydes phosphate dehydrogenase; 5-CCACCCATGGCAAATTCCATGGCA-3' and 5-TCTAGACGGGCAGGTCAGGTCCACC-3') and hBD-3 (5-TCTCAGCGTGGGGTGAAGC-3' and 5'CGGCCGCCTCTGACTCTG-3') were used. Primers were from TIB Molbiol (Berlin, Germany). After amplification, PCR products were separated by electrophoresis through a $2 \%$ agarose gels, stained with ethidium bromide and then visualized. GAPDH expression was used to confirm use of equal amounts of cDNA in each experiment.

\section{hBD-3 ELISA}

A549, SAEC and alveolar macrophages were infected as indicate, supernatants were collected. Samples were 10 times concentrated with Amicon Ultra-15 Centrifugal Filter Unit with Ultracel-3 membrane according to manufacturer instructions (Millipore $\mathrm{GmbH}$, Schwalbach, Germany) and processed for hBD-3 quantification by ELISA (Phoenix Pharmaceuticals Inc., Belmont, CA, USA). Since the probes were 10 times concentrated, we presented in the figures the concentration of hBD-3 corresponding to a non concentrated probe.

\section{Western Blot}

A549 cells were transfected or infected as indicated. Cells were lysed in buffer containing Triton X-100, subjected to SDS-PAGE and blotted on Hybond-ECL membrane (Amersham Biosciences, Freiburg, Germany). Immunodetection of target proteins was carried out with specific antibodies against p-JNK, JNK, c-Jun, $\beta$-actin (antibodies were purchased from Santa Cruz Biotechnologies, Santa Cruz, CA, USA) and p-c-Jun (Ser 73) (purchased from Cell Signaling, Frankfurt, Germany). In all experiments, $\beta$-actin was detected simultaneously to confirm equal protein load.

\section{Chromatin immunoprecipitation}

A549 cells were infected with L. pneumophila 130b. Cells were processed for chromatin immunoprecipitation
(ChIP) as described elsewhere [21]. $h b d-3$ promoter DNA was amplified by PCR using RedTaq polymerase (Qiagen, Hilden, Germany). PCR products were separated by agarose gel electrophoresis and detected by ethidium bromide staining of gels. Equal amounts of input DNA were confirmed by gel electrophoresis. For immunoprecipitation, the antibodies used were purchased from Santa Cruz Biotechnology (polymerase II, c-Jun), Frankfurt, Germany. The following promoter-specific primers for $h b d 3$ were used: sense 5'-TCCCAGAACTAACACACCCTT-3' and antisense 5'-TTCCAGCCACAGCTGCAATT-3'.

\section{Statistical methods}

Data are shown as means \pm SEM of at least three independent experiments. A one-way ANOVA test was used for data of figure 1D - F; Figure 2; Figure 3A and B; Figure $4 \mathrm{~A}$ and $4 \mathrm{C}$. The main effects were then compared by a Newman-Keuls' post-test. A two way ANOVA test was used for data of Figure 3C, D and 3E and Figure 5 and main effects were compared by a Bonferroni post-test. $\mathrm{P}$ $<0.05$ was considered to be significant and indicated by asterisks or H-Keys. If not indicated otherwise, test was performed vs. control $(*)$ or stimulated probe vs. inhibitor treated probe $(\#)$.

\section{Results}

L. pneumophila induced hBD-3 expression of human alveolar epithelial cells and macrophages

SAEC, A549 cells as well as primary human alveolar macrophages were infected with L. pneumophila strain $130 \mathrm{~b}$ or stimulated with TNF $\alpha$ as control for different periods of time and analyzed by RT-PCR and ELISA (Figure 1). An increased mRNA level of hBD-3 was observed in primary lung cells (SAEC) (figure 1A), in A549 cells (figure $1 B$ ) and in primary alveolar macrophages (figure 1C). Furthermore, a strong hBD-3 release could be demonstrated by ELISA in all three cell types infected with $L$. pneumophila (figure 3D - F). This suggests an important role of hBD-3 for the L. pneumophila-induced innate immune response of the lung. In A549, a time dependent increase of $\mathrm{hBD}-3$ release (figure $1 \mathrm{E}$ ) was observed. Next we addressed the mechanisms involved in L. pneumophila-induced hBD-3 expression by using A549 cells.

The type II and IV secretion systems of $L$. pneumophila are not essential for $\mathrm{hBD}-3$ release of alveolar epithelial cells

The type II (lsp) as well as the Dot/Icm type IVB secretion system is known to be important pathogen factors of L. pneumophila [3,4]. Infection of A549 cells with $L$. pneumophila strains JR32 and Corby induced comparable $\mathrm{hBD}-3$ release to strain $130 \mathrm{~b}$ (figure $1 \mathrm{~B}, 2$ ) suggesting that induction of this peptide is common in L. pneumophila infection of alveolar epithelium. Furthermore, no signifi- 


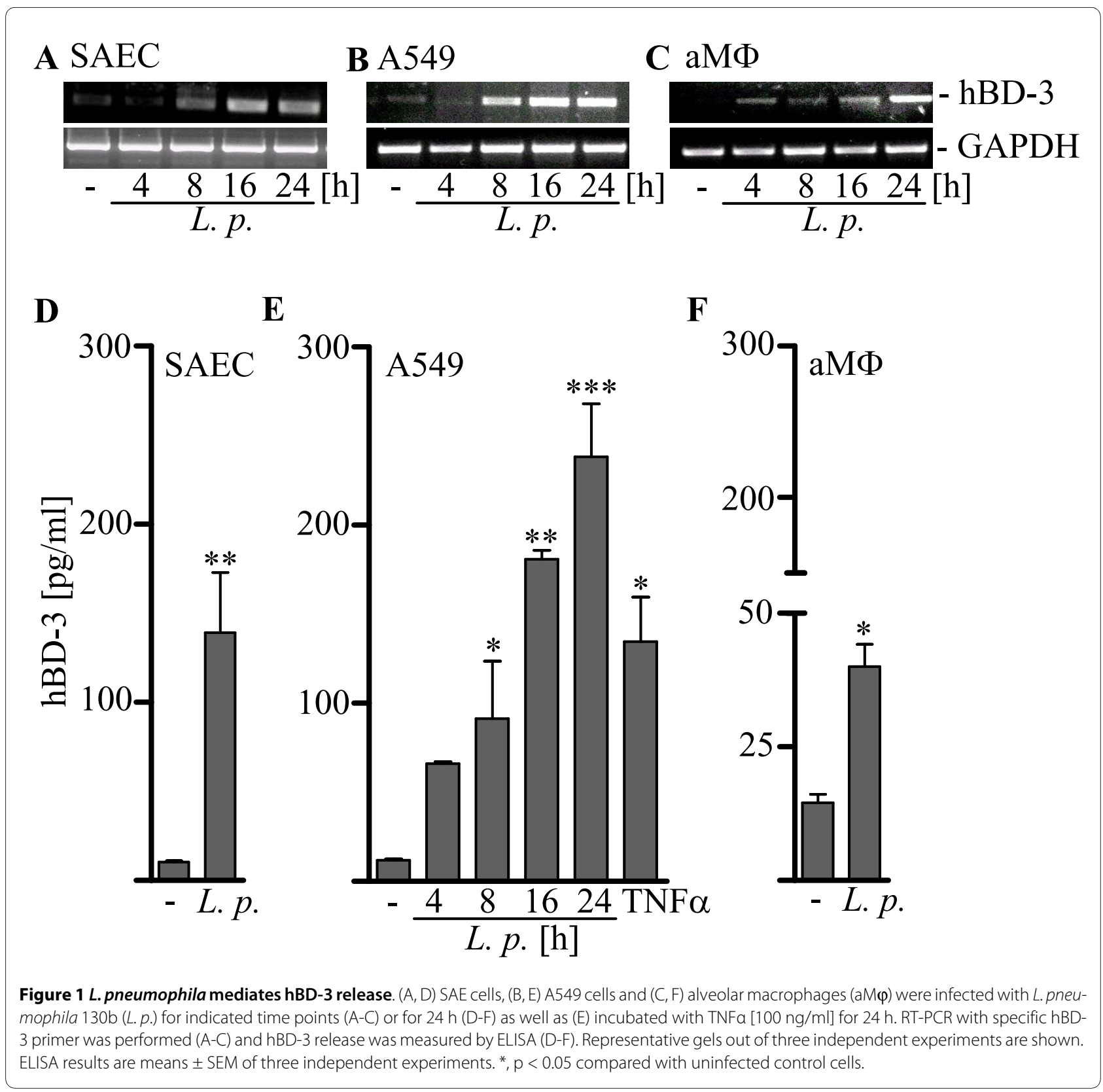

cant differences could be observed among the effects of JR32 $\Delta \operatorname{dot} A$ (figure 2) and Corby $\Delta l s p D E$ (figure 2) deletion mutants and wild-type strains with respect to hBD-3 liberation. These data suggest that the types II as well as the type IVB secretion system and their effector molecules are not involved in L. pneumophila-induced hBD-3 release. Furthermore, data indicates that intracellular replication of L. pneumophila appears not to be required for L. pneumophila-induced release of hBD-3, because the presence of the type IVB secretion system was shown not to be important.
hBD-3 release induced by $L$. pneumophila in alveolar epithelial cells is controlled by TLR2, TLR5, and TLR9

Following our previous and present observations that $L$. pneumophila strongly activates lung epithelial cells $[20,21,24]$, we tested the hypothesis that TLR2, TLR5, and TLR9 might be essential for the observed hBD-3 induction. To address this issue we stimulated SAEC with the TLR2 ligand Malp-2, the TLR5 ligand flagellin and non-methylated CpG-motifs (ODN) as ligand for TLR9. Incubation of SAEC with all agonists stimulated the release of hBD-3 (figure $3 \mathrm{~A}$ ). Furthermore we assessed the role of flagellin for the L. pneumophila induced hBD- 


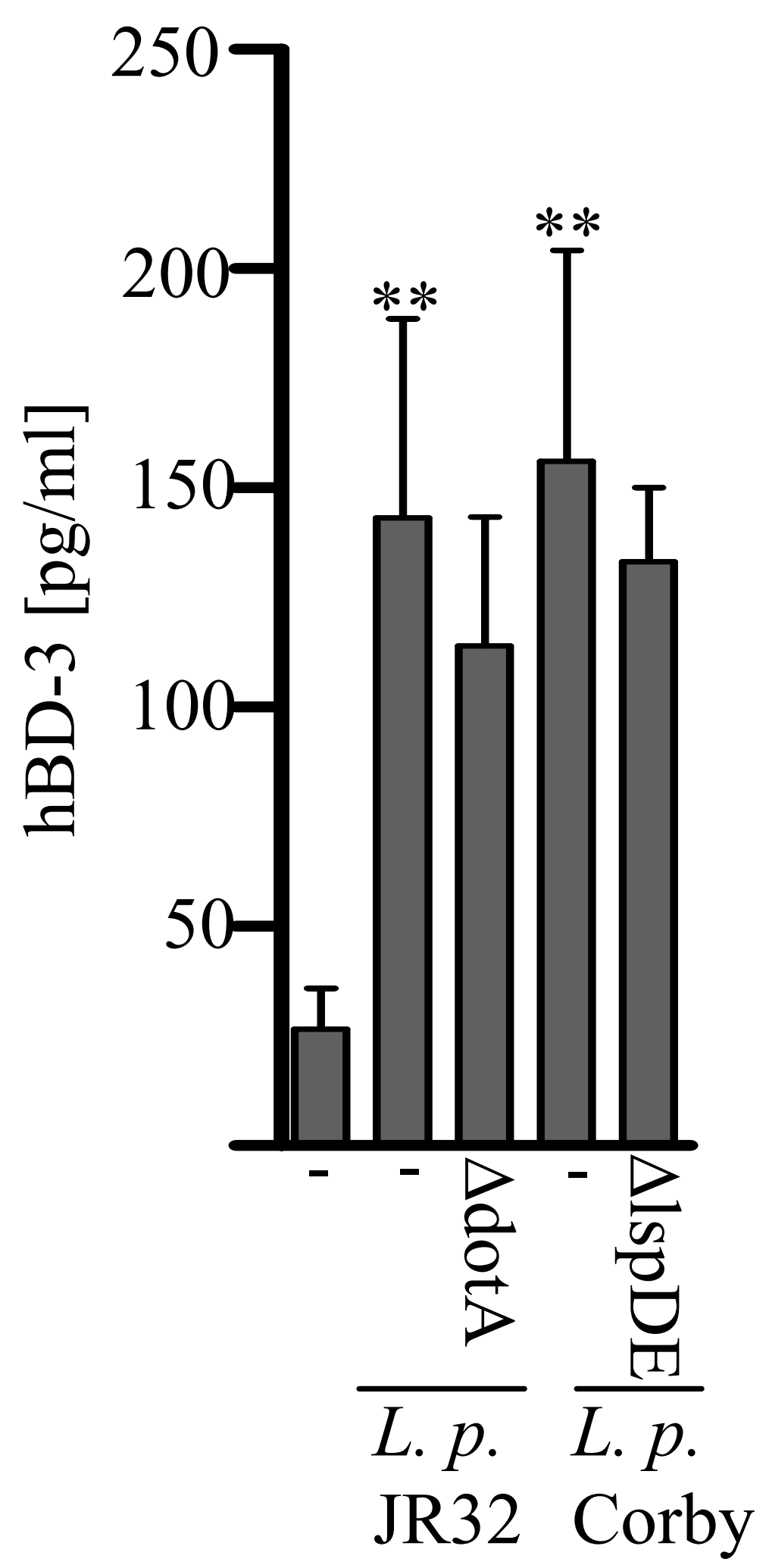

Figure $\mathbf{2}$ The type II and IVB secretion systems of $L$. pneumophila are not essential for hBD-3 release in A549 cells. A549 cells were infected with (A) L. pneumophila JR32, JR32 $\triangle$ dotA, and (B) Corby as well as Corby $\triangle$ /spDE for $24 \mathrm{~h}$. hBD-3 release was analyzed by ELISA. ELISA data presented are means \pm SEM of four separate experiments. ${ }^{*}, \mathrm{p}<0.05$ compared with uninfected control cells. 
A $\quad$ B

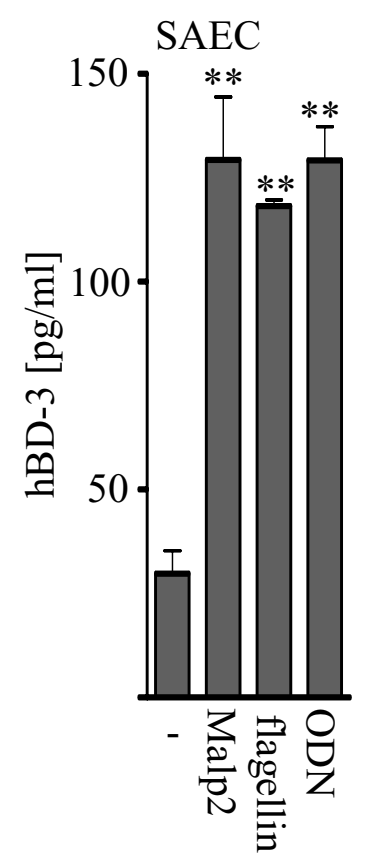

D

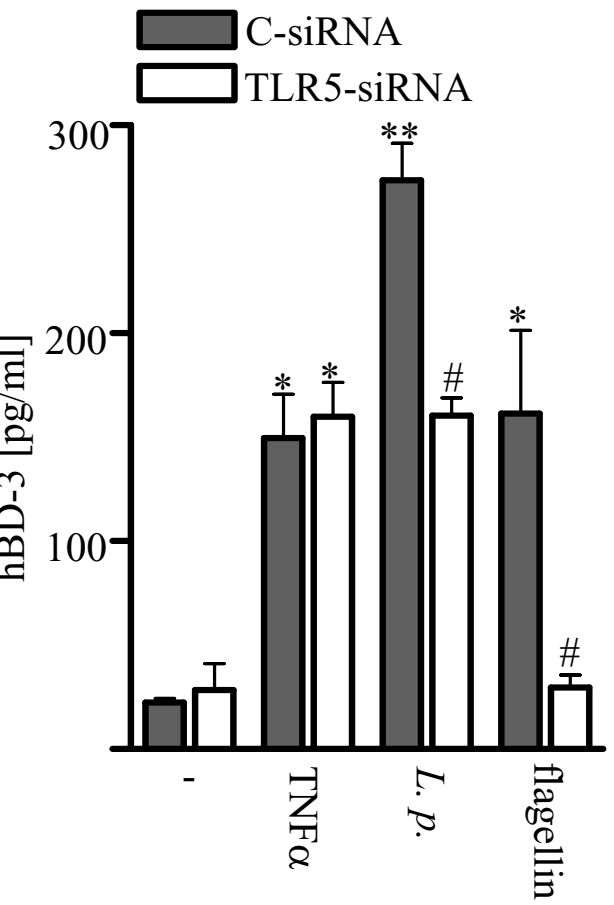

B

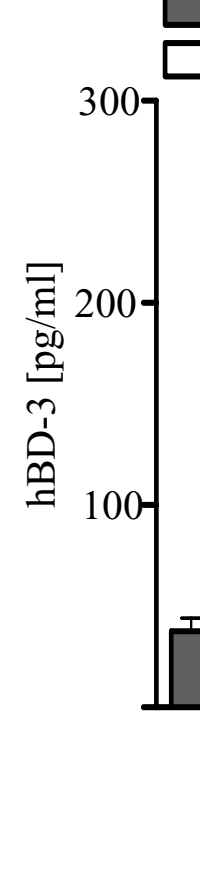

C-siRNA

TLR2-siRNA

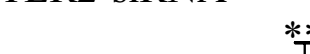<smiles>C1CCCCCC1</smiles>

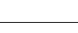

C

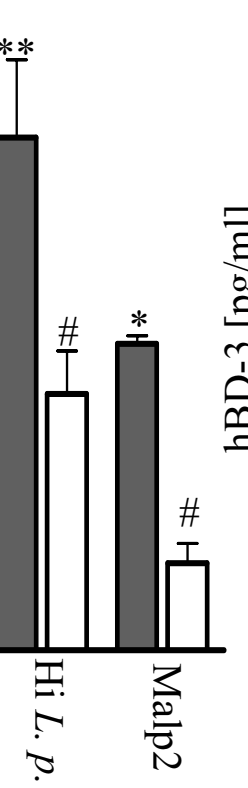

$\mathbf{E}$

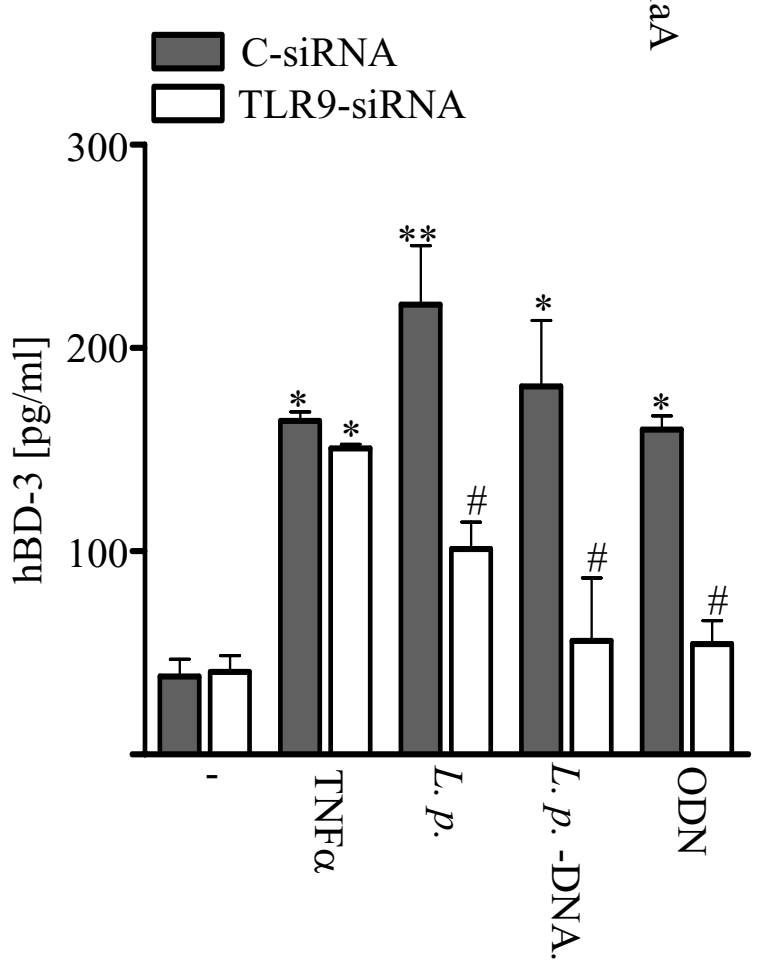

Figure 3 hBD-3 release induced by L. pneumophila in alveolar epithelial cells is controlled by TLR2, TLR5 and TLR9. (A) SAEC were incubated with the TLR2 ligand Mapl $2[1 \mu \mathrm{g} / \mathrm{ml}]$, purified flagellin to activte TLR5 [1 $\mathrm{\mu g} / \mathrm{ml}]$ and ODN as TLR9 ligand [1 $\mu \mathrm{M}]$. After $24 \mathrm{~h}$ hBD-3 release was measure by ELISA. RNAi experiments in A549 cells to inhibit expression of endogenous TLR2, TLR5 or TLR9 respectively were performed. Cells were incubated for $72 \mathrm{~h}$ with TLR2- and for $48 \mathrm{~h}$ with TLR5- and TLR9-specific siRNA as well as a non-silencing control siRNA (C-siRNA). Infection with L. pneumophila strain $130 \mathrm{~b}$ (L.p.), incubation with TNFa $[100 \mathrm{ng} / \mathrm{ml}]$ or (B) the TLR2 ligand Malp $2[1 \mu \mathrm{g} / \mathrm{ml}]$ and heat inactivated L. pneumophila, Corby, Corby $\triangle$ flaA or (C, D) incubated with flagellin [1 $\mu \mathrm{g} / \mathrm{ml}]$ and (E) the TLR9 ligand ODN [1 $\mu \mathrm{M}]$ and DNA [5 $\mu \mathrm{g} / \mathrm{ml}]$ from Legionella. After $24 \mathrm{~h} \mathrm{hBD-3}$ release was measure by ELISA. ELISA data presented are means \pm SEM of four separate experiments. ${ }^{*}, \mathrm{p}<0.05$ compared with uninfected control cells; \#, $\mathrm{p}<0.05$ control siRNA versus target siRNA. 


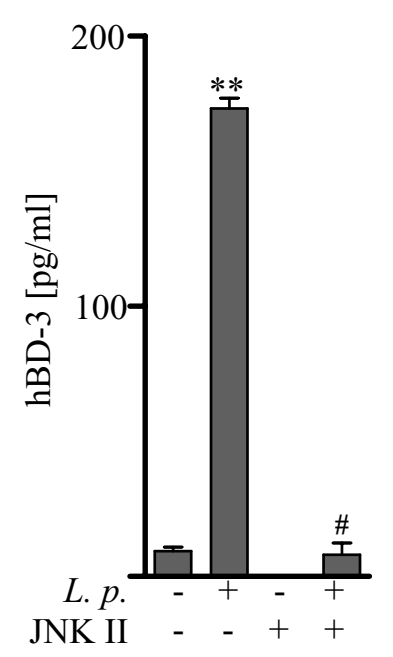

A

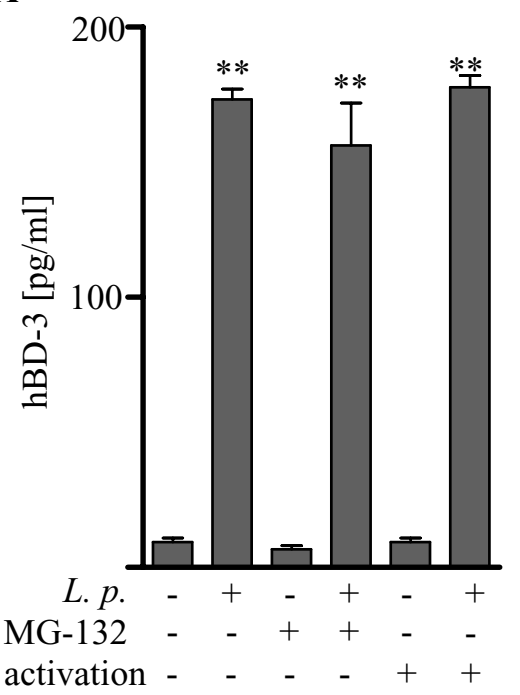

B

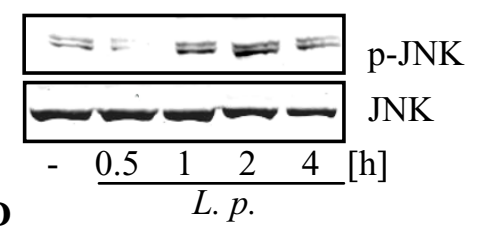

C NF- $\kappa \mathrm{B}$ activation - $\quad-\quad-\quad+\quad+$

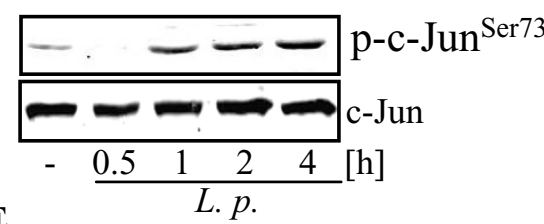

$\mathbf{E}$

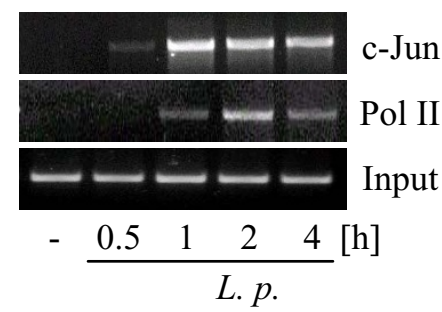

$$
\text { L. } p \text {. }
$$
inhibitor

Figure 4 hBD-3 expression in L. pneumophila - infected cells requires AP-1 (c-Jun). (A) A549 cells were pre-incubated with the proteasome in-

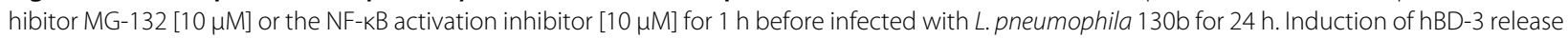
was measured by ELISA. (B) To elucidate the contribution of the JNK-AP-1 pathway, L. pneumophila mediated activation of JNK was detected time dependent by western blot analysis and (C) A549 cells were pre-incubated with the JNK inhibitor JNK II [10 $\mu \mathrm{M}]$ for $1 \mathrm{~h}$ before infected with L. pneumophila 130b for $24 \mathrm{~h}$. Induction of hBD-3 release was measured by ELISA. (D) c-Jun phosphorylation was assessed by western blotting after infection of A549 cells with L. pneumophila 130b at indicated time points. (E) Furthermore A549 cells were infected with L. pneumophila 130b at indicated time points and the binding patterns of Polymerase II (Pol II), and c-Jun to the hBD-3 promoter were analyzed by ChIP. Representative gels or blots out of three independent experiments are shown. ELISA data presented are means \pm SEM of three separate experiments. ${ }^{*}, p<0.05$ compared with uninfected control cells; \#, $p<0.05$ infected cells versus infected cells with specific inhibitors.

3 release in detail by infecting A549 cells with wild type Legionella as well as a flagellin-deficient mutant strain (Corby $\Delta f l a A$ ) (figure $3 \mathrm{~B})$. When comparing the wild type strain with a flagellin-deficient mutant (Corby $\triangle$ flaA $)$ significant difference in hBD-3 release could be observed (figure $3 \mathrm{~B}$ ), indicating that activation of TLR5 was important for hBD-3 secretion in L. pneumophila infected cells. To further examine the role of the TLRs we performed RNAi experiments in A549 cells to inhibit expression of endogenous TLR2, TLR5, and TLR9, respectively. We first confirmed that the TLR2-, TLR5- or TLR9-specific siRNA constructs but not the control siRNA resulted in the repression of protein levels in A549 cells (data not shown). A549 cells were transfected with TLR2-specific siRNA or control siRNA and were incubated with heat inactivated L. pneumophila, Malp-2 or infected with $L$. pneumophila 130b (figure 3C). Heat inactivated L. pneumophila induced hBD-3 release to the same extend as the viable L. pneumophila bacteria. Activation of TLR2 (with Malp-2) led to a similar hBD-3 release in A459 cells transfected with control siRNA. Reduced hBD-3 liberation could be observed in cells transfected with TLR2specific siRNA (figure 3C). Next we infected TLR5-specific siRNA-transfected A549 cells with L. pneumophila or incubated cells with flagellin and observed a decreased hBD-3 release mediated by depletion of TLR5 (figure 3D). Furthermore we addressed the role of TLR9 sensing nucleic acids in this process. Therefore A549 cells were transfected with specific TLR9-siRNA and afterwards incubated with purified L. pneumophila-DNA and ODN or infected with L. pneumophila (figure 3E). L. pneumophila-DNA and ODN strongly induced hBD-3 release to the same extent as the wild type strain and liberation of this peptide was reduced in all cells transfected with specific-TLR9-siRNA. In all RNAi experiments TNF $\alpha$ related hBD-3 production (figure 3) was not reduced. Overall, our observations suggest an important role for TLR2, TLR5 and TLR9 with respect to the hBD-3 induction observed in alveolar epithelium.

\section{hBD-3 expression in L. pneumophila - infected cells requires AP-1 (c-Jun)}

Recent studies showed that activation of NF- $\mathrm{kB}$ and/or AP-1 (c-Jun) controls hBD-3 expression [15-19]. Therefore, to further investigate a possible role of NF- $\mathrm{kB}$ activation for $L$. pneumophila-dependent hBD-3 release, we pre-incubated A549 cells with the proteasome inhibitor MG-132 to prevent IkB degradation. In addition we made 


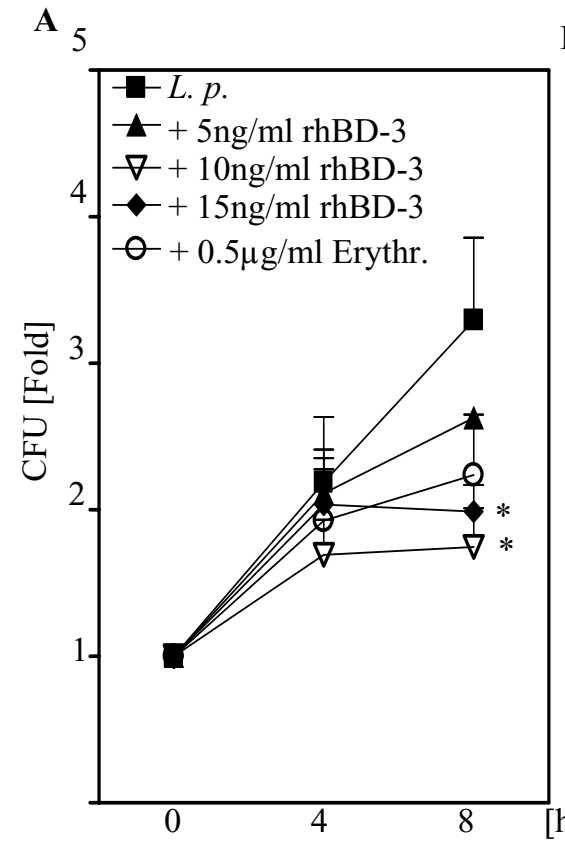

B

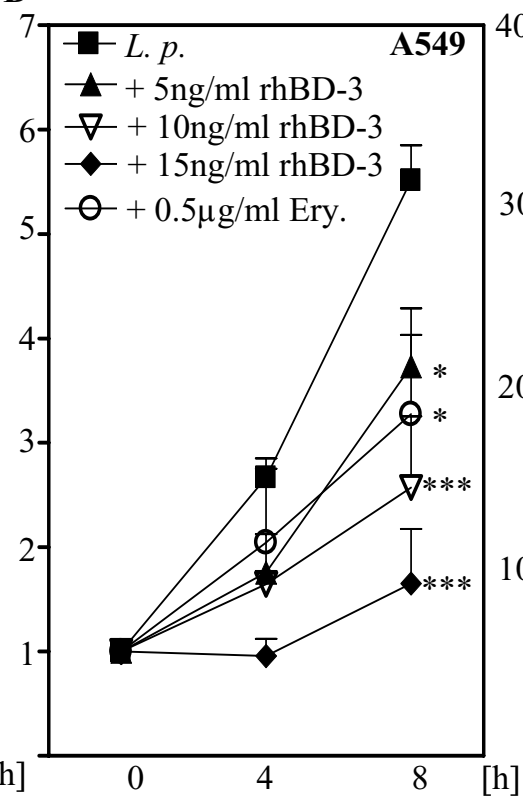

C

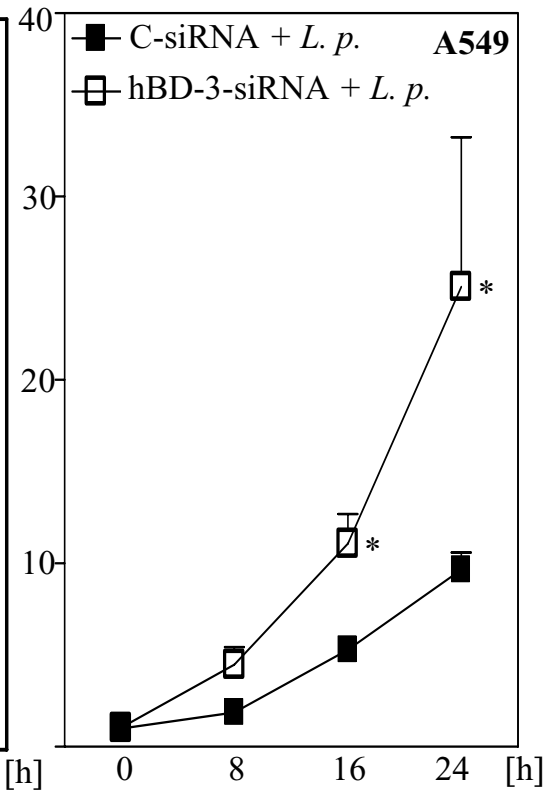

Figure 5 hBD-3 elicited a strong antimicrobial effect on L. pneumophila. (A) L. pneumophila 130b (L.p.) was incubated at $10^{5} \mathrm{cfu} / \mathrm{ml}$ in HAM's 12 with increasing concentrations of recombinant hBD-3 (rhBD-3) and erythromycin [erythr.; $0.5 \mathrm{\mu g} / \mathrm{ml}$ ] as indicated and multiplication of bacteria was assessed by colony forming units (cfu) assay plating solution mixture on agar plates after 4 and $8 \mathrm{~h}$. (B) A549 epithelial cells were infected with L. pneumophila 130b and replication assay was performed. (C) A549 epithelial cells were transfected with control siRNA (c-siRNA) or specific siRNA targeting hBD-3, and after $48 \mathrm{~h}$ infected with L. pneumophila 130b and intracellular replication of Legionella was assed by a replication assay after 8,16 and 24 h. Results are means \pm SEM of three independent experiments. ${ }^{*}, p<0.05$ compared with untreated control or control siRNA versus treated cells or target siRNA.

use of a NF- $\mathrm{kB}$ activity inhibiting peptide. Blocking IкB degradation by MG-132 or inhibiting NF- $\mathrm{kB}$ activity did not have any effect on hBD-3 release (figure 4A) in $L$. pneumophila-infected A549 cells whereas interleukin-8 release was significantly reduced (data not shown). Our data demonstrated that activation of NF-kB by L. pneumophila was not important for hBD-3 release in lung epithelium.

Activation of JNK is considered to participate in the regulation of inflammatory processes in alveolar epithelial cells [25]. Phosphorylation of the JNK kinase by $L$. pneumophila infection of epithelial cells was detected $1 \mathrm{~h}$ after infection, increased up to $2 \mathrm{~h}$, and decreased slightly at $4 \mathrm{~h}$ (figure $4 \mathrm{~B}$ ). Since JNK-mediated phosphorylation enhances the ability of c-Jun, a component of the AP-1 transcription factor, to activate transcription [20], we inhibited this kinase by pre-incubation of A549 cells with a JNK inhibitor (JNK II) before infection with L. pneumophila 130b. As shown in figure 4C, inhibition of JNK abolished L. pneumophila induced hBD-3 release. These observations suggest that JNK is important for the $L$. pneumophila-induced hBD-3 release in lung epithelial cells.
To further investigate the role of AP-1, we addressed the AP-1 subunit c-Jun activation following infection of alveolar epithelial cells with $L$. pneumophila. We observed a time dependently increased phosphorylation at serine 73 of the AP-1 subunit c-Jun (figure 4D).

To characterize the mechanism by which AP-1 (c-Jun) contributes to L. pneumophila-induced hBD-3 expression, the recruitment of c-Jun to the $h b d-3$ promoter were evaluated by ChIP assay. We observed an increase of the AP-1 subunit c-Jun binding to the $h b d-3$ promoter (figure $4 \mathrm{E})$ whereas the NF- $\mathrm{kB}$ subunit p65 was not recruited (data not shown). An increased binding of the RNA polymerase II (Pol II) to the $h b d-3$ promoter was indicative for the subsequent activation of the $h b d-3$ gene in infected A549 cells. These experiments confirm that the JNK-AP-1 (c-Jun) pathway controls hBD-3 expression in L. pneumophila infected alveolar epithelial cells.

The chemical inhibitors used in these experiments did neither reduce viability and proliferation of the A549 cells nor induces morphological signs of cytotoxicity, or alterations of bacterial growth within the time-frame tested (data not shown). 


\section{hBD-3 elicited a strong antimicrobial effect towards $L$. pneumophila}

To study the susceptibility of L. pneumophila to hBD-3, we incubated the wild type 130b in suspension with increasing concentrations of recombinant hBD-3 and a cfu assay was performed. As control for inhibition of replication we used the antibiotic erythromycin [26]. hBD-3 efficiently inhibited replication of this strain of Legionella in all used concentrations (figure 5A). Next we elucidated if hBD-3 has an antimicrobial effect towards intracellular Legionella growth. Therefore we infected A549 cells with L. pneumophila for a replication assay. Treatment with hBD-3 reduced the replication of the intracellular bacteria (figure 5B). Finally, the role of endogenous hBD-3 for intracellular replication of Legionella was tested in A549 cells transfected with hBD-3-specific siRNA or control siRNA. Knockdown of hBD-3 was confirmed by ELISA and RT-PCR (data not shown). The intracellular replication of L. pneumophila 130b was enhanced in cells transfected with specific hBD-3 siRNA compared to cells transfected with control siRNA (figure 5C), suggesting the importance of this peptide in Legionella-induced innate immune response.

\section{Discussion}

In the study presented, we demonstrate that L. pneumophila- induced hBD-3 expression was dependent on TLR2, TLR5 and TLR9 in human alveolar epithelial cells. The activation of JNK-pathway was identified to play a key role for L. pneumophila- triggered hBD-3 release. Furthermore, detailed analysis of signal transduction pathways provides evidence that activation of AP-1 subunit c-Jun but not NF-kB plays a key role in L. pneumophila- mediated $\mathrm{hBD}-3$ release (figure 6). We demonstrated also that hBD-3 elicited an antimicrobial effect towards L. pneumophila. We also showed that recombinant hBD-3 decreased replication of Legionella more efficient in lower concentrations than the antibiotic erythromycin used in treatment of Legionnaires' disease [26]. The mechanism by which defensins kill or inactivate bacteria is not precisely understood but is generally thought to be related to a perforation of the peripheral microbial membrane [12]. A recently published study showed a co-localisation of endogenous hBD-2 with the bacterial cell wall of extra- and intracellular replicating Mycobacterium tuberculosis in A549 cells [27]. For hBD-3 a similar antimicrobial mechanism can be assumed since a keratinocyte cell line engineered to overexpress hBD-3 demonstrated significant antimicrobial activity against Staphylococcus aureus [28]. On the other hand, hBD-3 can activate the NF- $\mathrm{KB}$ pathway via a TLR-triggered mechanisms [29]). This may induce secondary effector molecules which may reduce intracellular replication of Legionella. Since we observed in our study an antimicro- bial effect within four hours, we presume that hBD-3 kills L. pneumophila via direct perforation of the bacterial membrane.

The expression of hBD-3 in respiratory cells, especially in infections of the lung, is not well understood and was so far rather investigated in studies of oral infections as well as in epithelium of skin and intestine $[12,16,17,30,31]$. Our data showed that L. pneumophila infection of pulmonary epithelium and alveolar macrophages led to increased mRNA levels of hBD-3 and a strong secretion of this peptide. Since different isolates of L. pneumophila serogroup1 were found to induce a comparable release of $\mathrm{hBD}-3$, it is likely that $\mathrm{hBD}-3$ production is a common response of lung epithelial cells to $L$. pneumophila infection. This assumption is supported by a study which showed increased hBD-3 concentrations in respiratory tract and serum of patients suffering bacterial pneumonia [14]. In this study, hBD-3 exhibited a strong antibacterial effect on Staphylococcus aureus, Escherichia coli and Pseudomonas aeruginosa [14]. Since we also demonstrated that hBD-3 has an antimicrobial effect towards L. pneumophila and this peptide do orchestrate the recruitment of alveolar macrophages to the site of infection [32], we assume that this defensin might be important for immune response in infectious diseases of the lung.

Pulmonary epithelial cells may detect L. pneumophila by TLRs [5-7]. In accordance we demonstrated that recognition of $L$. pneumophila by TLR2, TLR5 and TLR9 was essential for the production of hBD-3. In recently published studies, it was shown that hBD-3 expression was induced TLR2-dependent in skin epithelial cells $[16,33,34]$. To our knowledge, our study showed for the first time the induction of hBD-3 via activation of TLR5 and TLR9.

In mice pneumonia studies, TLR2, TLR5 and TLR9 were required for effective innate immune responses against $L$. pneumophila $[5,7,35]$. Since we demonstrated that inhibition of all three TLRs decreases L. pneumophila-induced hBD-3 release, we assume that these receptors might be essential for antimicrobial innate immune response in Legionella infections. Interestingly, hBD-3 liberation was not reduced in infections with type II or IVB secretion system mutant strains, suggesting that recognition of bacterial membrane component via TLR2, recognition of Legionella-flagellin via TLR5 and/or nonmethylated bacterial DNA through TLR9 might be the major pathways for L. pneumophila induced hBD-3.

A complex signaling network regulates the expression of inducible hBD-3 [16,17,33]. In $L$. pneumophilainfected lung epithelial cells, we noted that the bacteria induced a JNK-dependent release of hBD-3. This prompted to the assumption that JNK related transcription factor AP-1 is involved in this process. Indeed, we 


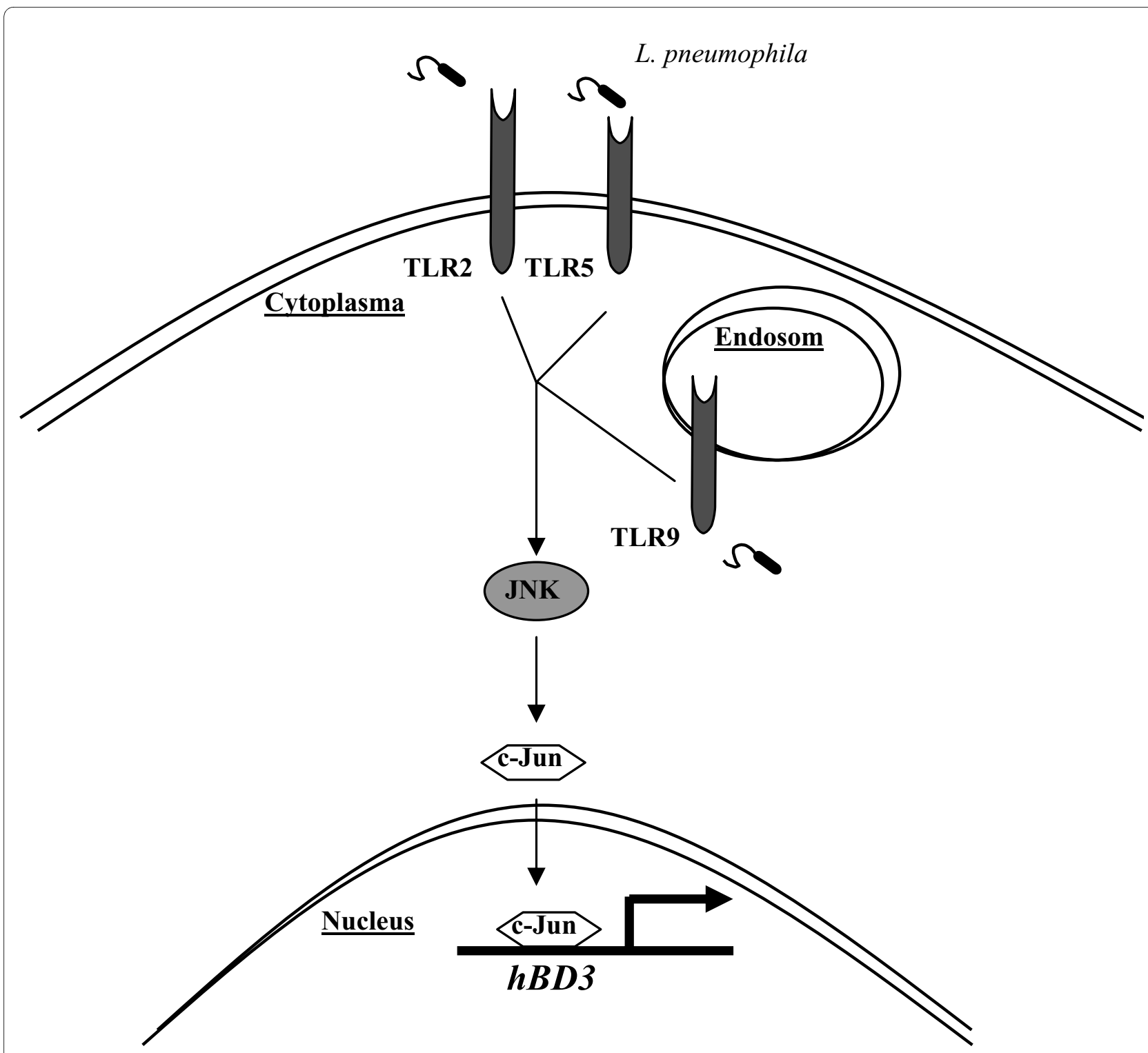

Figure 6 Molecular scheme of Legionella pneumophila-induced hBD-3 expression. L. pneumophila activates TLR2, TLR5, and TLR9. Downstream signaling leads to the activation of JNK with subsequent recruitment of AP-1 subunit c-jun to the hBD-3 promoter leading to hBD-3 expression.

showed that AP-1 subunit c-Jun was recruited to $h b d-3$ promoter. Our data are in line with previous studies which demonstrated a strong AP-1 dependency of hBD-3 induction $[16,17]$. In contrast, a recently reported role of $\mathrm{NF}-\mathrm{kB}$ in the regulation of the $h B D-3$ gene in keratinocytes has been published [15]. Since an NF- $k B$ binding site is also located on $h b d-3$ promoter, we tested if a binding of this transcription factor could be observed in $L$. pneumophila- infected alveolar epithelial cells and failed to confirm a binding of NF-kB subunit p65 to this structure (data not shown). This strengthens our data that mainly AP-1 was essential for L. pneumophila induced release of hBD-3 in pulmonary cells.
Functional studies and signal transduction experiments of this study were performed in A549 cells. Since infection with L. pneumophila and stimulation with TLR agonists showed comparable expression of hBD-3 in A549 cells and primary SAEC, we assume similarities in regulation of hBD-3 between both cell types. Furthermore, previous studies by our and other groups demonstrated analogous behavior of A549 cells and the primary SAEC in release of cytokines, defensins, prostaglandin E2 as well as in production of reactive oxygen species after infection $[20,21,24,36,37]$. Nevertheless, A549 cells are a tumor cell line and further studies in vivo are needed to dissect the signaling pathways mediating L. pneumophila-related defensin release. 
In conclusion, we found that L. pneumophila-triggered hBD-3 release is TLR2, TLR5- and TLR9-dependent hBD-3 in human pulmonary epithelial cells. Expression of hBD-3 included activation of the JNK- AP-1 (c-Jun) pathway, whereas NF- $\mathrm{kB}$ was not essential for this process in A549 cells. Since control of the immune response is crucial to assure bacterial clearance and to prevent excessive tissue damage in pneumonia, the mechanism described above could be important for the host defense in Legionnaires' disease.

\section{Competing interests}

The authors declare that they have no competing interests.

\section{Authors' contributions}

PDN planned the project; SS and PDN designed and directed the experiments; SS carried out many of the experiments. KV and FL helped to carry out experiments. AF and $\mathrm{KH}$ provided advices for the experiments concerning Legionella pneumophila. SS and PDN analyzed the data and wrote the manuscript. BS, BO, $\mathrm{SH}, \mathrm{NS}$ provided advice for the experiments, the data analysis and helped to write the manuscript. NS and PDN supervised the research and contributed to manuscript criticisms. All authors read and approved the final manuscript.

\section{Acknowledgements}

The excellent technical assistance of Frauke Schreiber, Jacqueline Hellwig, Doris Stoll, Annika Kühn (Department of Internal Medicine/Infectious Diseases and Pulmonary Medicine, Berlin), and Kerstin Rydzewski (Robert Koch Institut, Berlin) is greatly appreciated. Part of this work will be included in the doctoral thesis of Stefanie Scharf. This work was supported by the German Research Foundation (DFG-SFB TRR84 to PD.N. and N.S.), the Bundesministerium für Bildung und Forschung (BMBF-FORSYS-Partner to B.S., BMBF Network PROGRESS to S.H. and N.S.), and the Charité-Universitätsmedizin Berlin (Scholarship to S.S).

\section{Author Details}

'Department of Internal Medicine/Infectious Diseases and Pulmonary Medicine, Charité - Universitätsmedizin Berlin, Germany, ${ }^{2}$ FORSYS Junior Research Group, Systems Biology of Lung Inflammation, Charité-

Universitätsmedizin Berlin, Germany, ${ }^{3}$ FG1 1 Division of Bacterial infections, Robert Koch-Institut, Wernigerode, Germany and 4 P26 Nosocomial Infections of the Elderly, Robert Koch-Institut, Berlin, Germany

Received: 15 January 2010 Accepted: 8 July 2010

Published: 8 July 2010

\section{References}

1. von Baum H, Ewig S, Marre R, Suttorp N, Gonschior S, Welte T, Luck C: Community-acquired Legionella pneumonia: new insights from the German competence network for community acquired pneumonia. Clin Infect Dis 2008, 46:1356-1364. 4

2. Hippenstiel S, Opitz B, Schmeck B, Suttorp N: Lung epithelium as a sentinel and effector system in pneumonia--molecular mechanisms of pathogen recognition and signal transduction. Respir Res 2006, 7:97.

3. Cianciotto NP: Many substrates and functions of type II secretion: lessons learned from Legionella pneumophila. Future Microbio/ 2009, 4:797-805.

4. Isberg RR, O'Connor TJ, Heidtman M: The Legionella pneumophila replication vacuole: making a cosy niche inside host cells. Nat Rev Microbiol 2009, 7:13-24. 24

5. Bhan U, Trujillo G, Lyn-Kew K, Newstead MW, Zeng X, Hogaboam CM, Krieg AM, Standiford TJ: Toll-like receptor 9 regulates the lung macrophage phenotype and host immunity in murine pneumonia caused by Legionella pneumophila. Infect Immun 2008, 76:2895-2904.

6. Girard RF, Pedron TF, Uematsu VS, Balloy VF, Balloy MF, Chignard R, Akira S, Chaby R: Lipopolysaccharides from Legionella and Rhizobium stimulate mouse bone marrow granulocytes via Toll-like receptor 2 . $J$ Cell Sci 2003, 116:293-302.

7. Hawn TR, Verbon A, Lettinga DK, Zhao LP, Li SS, Laws RJ, Skerrett SJ, Beutler B, Schroeder L, Nachman A, Ozinsky A, Smith KD, Aderem A: A common dominant TLR5 stop codon polymorphism abolishes flagellin signaling and is associated with susceptibility to legionnaires' disease. J Exp Med 2003, 198:1563-1572.

8. Hiemstra PS: The role of epithelial beta-defensins and cathelicidins in host defense of the lung. Exp Lung Res 2007, 33:537-542. 47

9. McCray PB Jr, Bentley L: Human airway epithelia express a betadefensin. Am J Respir Cell Mol Biol 1997, 16:343-349.

10. Bals R, Wang X, Z Wu, Freeman T, Bafna V, Zasloff M, Wilson JM: Human beta-defensin 2 is a salt-sensitive peptide antibiotic expressed in human lung. J Clin Invest 1998, 102:874-880

11. Harder J, Bartels J, Christophers E, Schroder JM: A peptide antibiotic from human skin. Nature 1997, 387:861.

12. Harder J, Bartels J, Christophers E, Schroder JM: Isolation and characterization of human beta -defensin-3, a novel human inducible peptide antibiotic. J Biol Chem 2001, 276:5707-5713.

13. Maisetta G, Batoni G, Esin S, Florio W, Bottai D, Favilli F, Campa M: In vitro bactericidal activity of human beta-defensin 3 against multidrugresistant nosocomial strains. Antimicrob Agents Chemother 2006, 50:806-809

14. Ishimoto H, Mukae H, Date Y, Shimbara T, Mondal MS, Ashitani J, Hiratsuka T, Kubo S, Kohno S, Nakazato M: Identification of hBD-3 in respiratory tract and serum: the increase in pneumonia. Eur Respir J 2006, 27:253-260

15. Albanesi C, Fairchild HR, Madonna S, Scarponi C, De Pita O, Leung DY, Howell MD: IL-4 and IL-13 negatively regulate TNF-alpha- and IFNgamma-induced beta-defensin expression through STAT-6, suppressor of cytokine signaling (SOCS)-1, and SOCS-3. J Immunol 2007, 179:984-992.

16. Menzies BE, Kenoyer A: Signal transduction and nuclear responses in Staphylococcus aureus-induced expression of human beta-defensin 3 in skin keratinocytes. Infect Immun 2006, 74:6847-6854.

17. Steubesand N, Kiehne K, Brunke G, Pahl R, Reiss K, Herzig KH, Schubert S, Schreiber S, Folsch UR, Rosenstiel P, Arlt A: The expression of the betadefensins $h B D-2$ and $h B D-3$ is differentially regulated by NF-kappaB and MAPK/AP-1 pathways in an in vitro model of Candida esophagitis. BMC Immunol 2009, 10:36.

18. Ventura JJ, Kennedy NJ, Lamb JA, Flavell RA, Davis RJ: c-Jun NH(2)terminal kinase is essential for the regulation of AP- 1 by tumor necrosis factor. Mol Cell Biol 2003, 23:2871-2882.

19. Lippmann J, Rothenburg S, Deigendesch N, Eitel J, van Meixenberger KLV, Slevogt H, N'Guessan PD, Hippenstiel S, Chakraborty T, Flieger A, Suttorp $\mathrm{N}$, Opitz B: IFNbeta responses induced by intracellular bacteria or cytosolic DNA in different human cells do not require ZBP1 (DLM-1/ DAI). Cell Microbio/ 2008, 10:2579-2588.

20. N'Guessan PD, Etouem MO, Schmeck B, Hocke AC, Scharf S, Vardarova K, Opitz B, Flieger A, Suttorp N, Hippenstiel S: Legionella pneumophilainduced PKCalpha-, MAPK-, and NF-kappaB-dependent COX-2 expression in human lung epithelium. Am J Physiol Lung Cell Mol Physiol 2007, 292:L267-L277.

21. Schmeck B, Lorenz J, N'Guessan PD, Opitz B, van LV, Zahlten J, Slevogt H, Witzenrath M, Flieger A, Suttorp N, Hippenstiel S: Histone acetylation and flagellin are essential for Legionella pneumophila-induced cytokine expression. J Immunol 2008, 181:940-947.

22. Edelstein PH: Improved semiselective medium for isolation of Legionella pneumophila from contaminated clinical and environmental specimens. J Clin Microbio/ 1981, 14:298-303. 578

23. Islam D, Bandholtz L, Nilsson J, Wigzell H, Christensson B, Agerberth B, Gudmundsson G: Downregulation of bactericidal peptides in enteric infections: a novel immune escape mechanism with bacterial DNA as a potential regulator. Nat Med 2001, 7:180-185.172

24. Scharf S, Hippenstiel S, Flieger A, Suttorp N, N'Guessan PD: Induction of human \{beta\}-Defensin-2 in pulmonary epithelial cells by Legionella pneumophila: Involvement of TLR2 and TLR5, p38 MAPK, JNK, NF\{kappa\}B and AP-1. Am J Physiol Lung Cell Mol Physiol 2010, 298:687-695.

25. Li W, Zheng S, Tang C, Zhu Y, Wang X: JNK-AP-1 pathway involved in interleukin-1 beta-induced calcitonin gene-related peptide secretion in human type II alveolar epithelial cells. Peptides 2007, 28:1252-1259.

26. Sabria M, Pedro-Botet ML, Gomez J, Roig J, Vilaseca B, Sopena N, Banos V: Fluoroquinolones vs macrolides in the treatment of Legionnaires disease. Chest 2005, 128:1401-1405.

27. Rivas-Santiago B, Schwander SK, Sarabia C, Diamond G, Klein-Patel ME, Hernandez-Pando R, Ellner JJ, Sada E: Human \{beta\}-defensin 2 is 
expressed and associated with Mycobacterium tuberculosis during infection of human alveolar epithelial cells. Infect Immun 2005, 73:4505-4511.

28. Sawamura D, Goto M, Shibaki A, Akiyama M, McMillan JR, Abiko Y, Shimizu $\mathrm{H}$ : Beta defensin-3 engineered epidermis shows highly protective effect for bacterial infection. Gene Ther 2005, 12:857-861.

29. Funderburg N, Lederman MM, Feng Z, Drage MG, Jadlowsky J, Harding CV, Weinberg A, Sieg SF: Human -defensin-3 activates professional antigen-presenting cells via Toll-like receptors 1 and 2. Proc Natl Acad SciUSA 2007, 104:18631-18635.

30. Fahlgren A, Hammarstrom S, Danielsson A, Hammarstrom ML: betaDefensin-3 and -4 in intestinal epithelial cells display increased mRNA expression in ulcerative colitis. Clin Exp Immunol 2004, 137:379-385.

31. Lu Q, Samaranayake LP, Darveau RP, Jin L: Expression of human betadefensin-3 in gingival epithelia. J Periodontal Res 2005, 40:474-481

32. Kawsar HI, Weinberg A, Hirsch SA, Venizelos A, Howell S, Jiang B, Jin G: Overexpression of human beta-defensin- 3 in oral dysplasia: potential role in macrophage trafficking. Oral Oncol 2009, 45:696-702. 2

33. Buchau AS, Schauber J, Hultsch T, Stuetz A, Gallo RL: Pimecrolimus enhances TLR2/6-induced expression of antimicrobial peptides in keratinocytes. J Invest Dermatol 2008, 128:2646-2654.

34. Sumikawa YF, Asada HF, Hoshino KF, Azukizawa HF, Katayama IF, Akira S, Itami S: Induction of beta-defensin 3 in keratinocytes stimulated by bacterial lipopeptides through toll-like receptor 2. Microbes Infect 2(8):1513-1521

35. Fuse ET, Tateda K, Kikuchi Y, Matsumoto T, Gondaira F, Azuma A, Kudoh S, Standiford TJ, Yamaguchi K: Role of Toll-like receptor 2 in recognition of Legionella pneumophila in a murine pneumonia model. JMed Microbiol 2007, 56:305-312.

36. Kao YJ, Piedra PA, Larsen GL, Colasurdo GN: Induction and regulation of nitric oxide synthase in airway epithelial cells by respiratory syncytial virus. Am J Respir Crit Care Med 2001, 163:532-539.

37. Vardarova K, Scharf S, Lang F, Schmeck B, Opitz B, Eitel J, Hocke AC, Slevogt H, Flieger A, Hippenstiel S, Suttorp N, N'Guessan PD: PKC(alpha) and PKC(epsilon) differentially regulate Legionella pneumophilainduced GM-CSF. Eur Respir J 2009, 34:1171-1179.

doi: 10.1186/1465-9921-11-93

Cite this article as: Scharf et al., Legionella pneumophila induces human beta Defensin-3 in pulmonary cells Respiratory Research 2010, 11:93

\section{Submit your next manuscript to BioMed Central} and take full advantage of:

- Convenient online submission

- Thorough peer review

- No space constraints or color figure charges

- Immediate publication on acceptance

- Inclusion in PubMed, CAS, Scopus and Google Scholar

- Research which is freely available for redistribution

Submit your manuscript at www.biomedcentral.com/submit
C Biomed Central 